\title{
Diagnostic Challenge of Diffusion Tensor Imaging in a Patient With Hemiplegia After Traumatic Brain Injury
}

\author{
Hye Eun Shin, $\mathrm{MD}^{1}$, Hoon Chang Suh, $\mathrm{MD}^{1}$, Si Hyun Kang, $\mathrm{MD}, \mathrm{PhD}^{1}$, \\ Kyung Mook Seo, MD, PhD ${ }^{1}$, Don-Kyu Kim, MD, PhD ${ }^{1}$, Hae-Won Shin, MD, PhD ${ }^{2}$
}

Departments of ${ }^{1}$ Physical Medicine and Rehabilitation, ${ }^{2}$ Neurology, Chung-Ang University College of Medicine, Seoul, Korea

\begin{abstract}
A 51-year-old man showed hemiplegia on his right side after a traumatic brain injury (TBI). On initial brain computed tomography (CT) scan, an acute subdural hemorrhage in the right cerebral convexity and severe degrees of midline shifting and subfalcine herniation to the left side were evident. On follow-up brain magnetic resonance imaging (MRI), there were multiple microhemorrhages in the left parietal and occipital subcortical regions. To explain the occurrence of right hemiplegia after brain damage which dominantly on the right side of brain, we used diffusion tensor imaging (DTI) to reconstruct the corticospinal tract (CST), which showed nearly complete injury on the left CST. We also performed motor-evoked potentials, and stimulation of left motor cortex evoked no response on both sides of upper extremity. We report a case of patient with hemiplegia after TBI and elucidation of the case by DTI rather than CT and MRI.
\end{abstract}

Keywords Diffusion tensor imaging, Traumatic brain injuries, Corticospinal tract

\section{INTRODUCTION}

After traumatic brain injury (TBI), cerebral hemorrhage and diffuse axonal injury (DAI) could coexist, and this could induce varying degrees of physical disability. And it is important to identify the extent of axonal injury early; however, this was a diagnostic challenge with conventional imaging tools $[1,2]$.
Although conventional magnetic resonance imaging (MRI) became one of the most powerful tools for providing structural information after TBI such as hemorrhage and herniation, additional information about axonal injuries was limited [2,3]. A new MRI technique known as diffusion tensor imaging (DTI) can reveal axonal organization of the brain, such as the corticospinal tract (CST), which was not possible with conventional imaging

Received March 11, 2016; Accepted June 20, 2016

Corresponding author: Si Hyun Kang

Department of Physical Medicine and Rehabilitation, Chung-Ang University Hospital, Chung-Ang University College of Medicine, 224-1 Heuksukdong, Dongjak-gu, Seoul 06974, Korea. Tel: +82-2-6299-1883, Fax: +82-2-6298-1866, E-mail: sihyun92@cau.ac.kr

ORCID: Hye Eun Shin (http://orcid.org/0000-0003-4903-9283); Hoon Chang Suh (http://orcid.org/0000-0001-5333-8986); Si Hyun Kang (http://orcid. org/0000-0003-0205-067X); Kyung Mook Seo (http://orcid.org/0000-0002-9762-085X); Don-Kyu Kim (http://orcid.org/0000-0001-8619-830X); HaeWon Shin (http://orcid.org/0000-0001-6050-7733).

(c) This is an open-access article distributed under the terms of the Creative Commons Attribution Non-Commercial License (http://creativecommons.org/ licenses/by-nc/4.0) which permits unrestricted noncommercial use, distribution, and reproduction in any medium, provided the original work is properly cited. Copyright (c) 2017 by Korean Academy of Rehabilitation Medicine 
studies. DTI provides information about the degree and direction of water diffusion in multiple directions within individual voxels of the MRI. The water diffusion data at the level of each individual voxel in a region can be summarized and visualized as an apparent diffusion coefficient (ADC) and fractional anisotropy (FA). Diffusion tensor tractography (DTT) is a method used to identify and visualize a continuous 3-dimensional trajectory by sequentially piecing together the estimates of fiber orientation from the directionality of individual voxels. It allows us to monitor the longitudinal evolution of axonal injuries and the efficacy of interventions in a variety of disease models [4]. DTI is now becoming widely available in clinical scanners, and human clinical studies are actively being performed employing this technique.

\section{CASE REPORT}

A 51-year-old man was admitted through the emergency room with decreased mental status on October 16, 2014. On admission, we could not check manual muscle test because of the patient's stuporous mental status; his Glasgow Coma Scale (GCS) score was 3. Brain computed tomography (CT) revealed an acute subdural hemorrhage in right cerebral convexity and a small subarachnoid hemorrhage in fronto-parietal sulci and sylvian cistern, with severe degrees of midline shifting and subfalcine herniation towards the left (Fig. 1). The patient was admitted to the Department of Neurosurgery and had decompressive craniectomy on the day of admission. On November 24, 2014, he had cranioplasty and additional craniotomy, and was transferred to the rehabilitation de- partment on December 11, 2014. The patient's GCS score was 14 at this time, and he could obey 2-step commands. Physical examination revealed motor weakness ranging from trace to fair grade on his right side with sensory deficits, whereas his left side showed minimal subjective weakness (grade 4). However, his deep tendon reflex was hyperactive at not only right upper and lower extremities but also left upper extremity. Spasticity appeared bilaterally; the right side was Modified Ashworth Scale grade 2, compared with the left side, which was grade 1 . In addition, pathologic reflex was observed bilaterally, indicated by a positive Hoffman sign on the left side and a Babinski sign and an ankle clonus on the right side.

Conventional MRI showed cytotoxic edema in right frontal and temporal lobe with hemorrhagic cortical contusions in right inferior frontal, temporal, and left parietal lobe. Multiple microhemorrhages in left parietal and occipital subcortical regions were observed in susceptibilityweighted images (Fig. 2). With these results, we could not completely explain the occurrence of hemiplegia on right side along with relatively mild weakness on the left side. We performed a brain MRI, adding DTI to reconstruct his CST using Philips Achieva 3.0T TX (Philips, Amsterdam, Netherlands). The imaging parameters were: field of view $250 \times 250, \mathrm{TR}=9213.90 \mathrm{~ms}, \mathrm{TE}=70.0 \mathrm{~ms}, 2.0-\mathrm{mm}$ thickness, and 1.0 number of excitations (NEX). The imaging data sets were transferred to a workstation (Philips Extended MR WorkSpace 2.6.3.4) to reconstruct the DTT.

The results showed that right CST was injured in the subcortical white matter, and left CST was nearly completely injured (Fig. 3). These findings were in correlation with the patient's symptoms, e.g., hemiplegia on the right
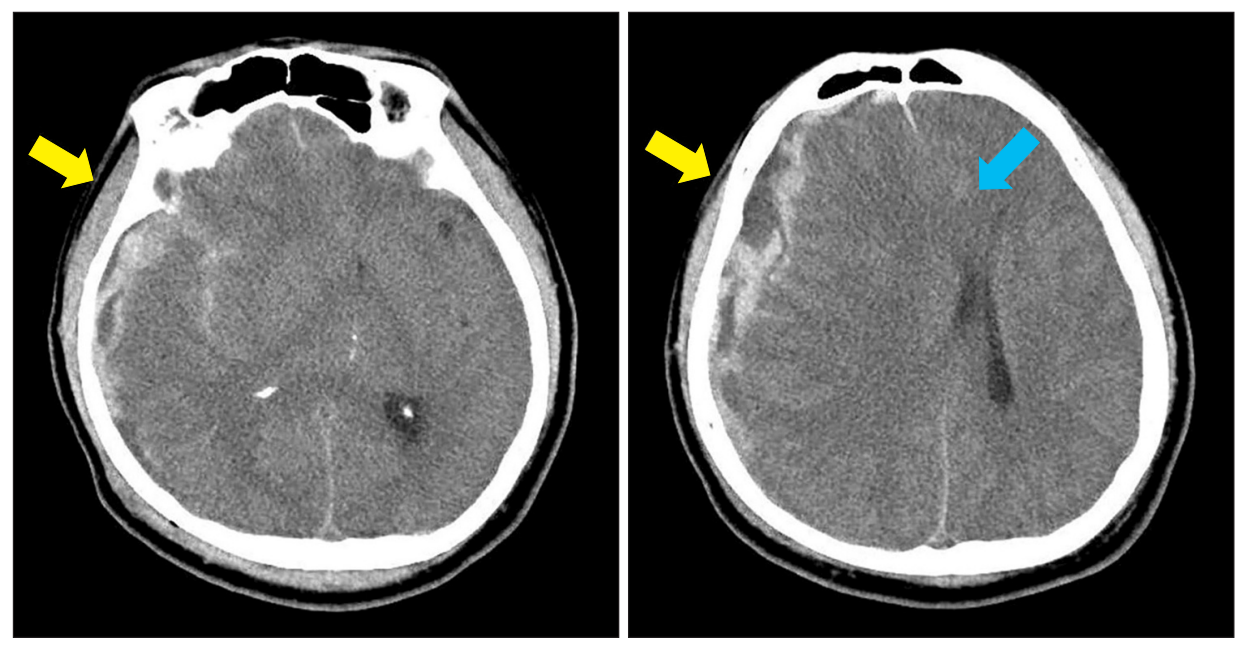

Fig. 1. Brain computed tomography at the onset of injury shows an acute subdural hemorrhage in right cerebral convexity and a small subarachnoid hemorrhage in frontoparietal sulci and sylvian cistern (yellow arrow), with severe degrees of midline shifting and subfalcine herniation towards the left side (blue arrow). 

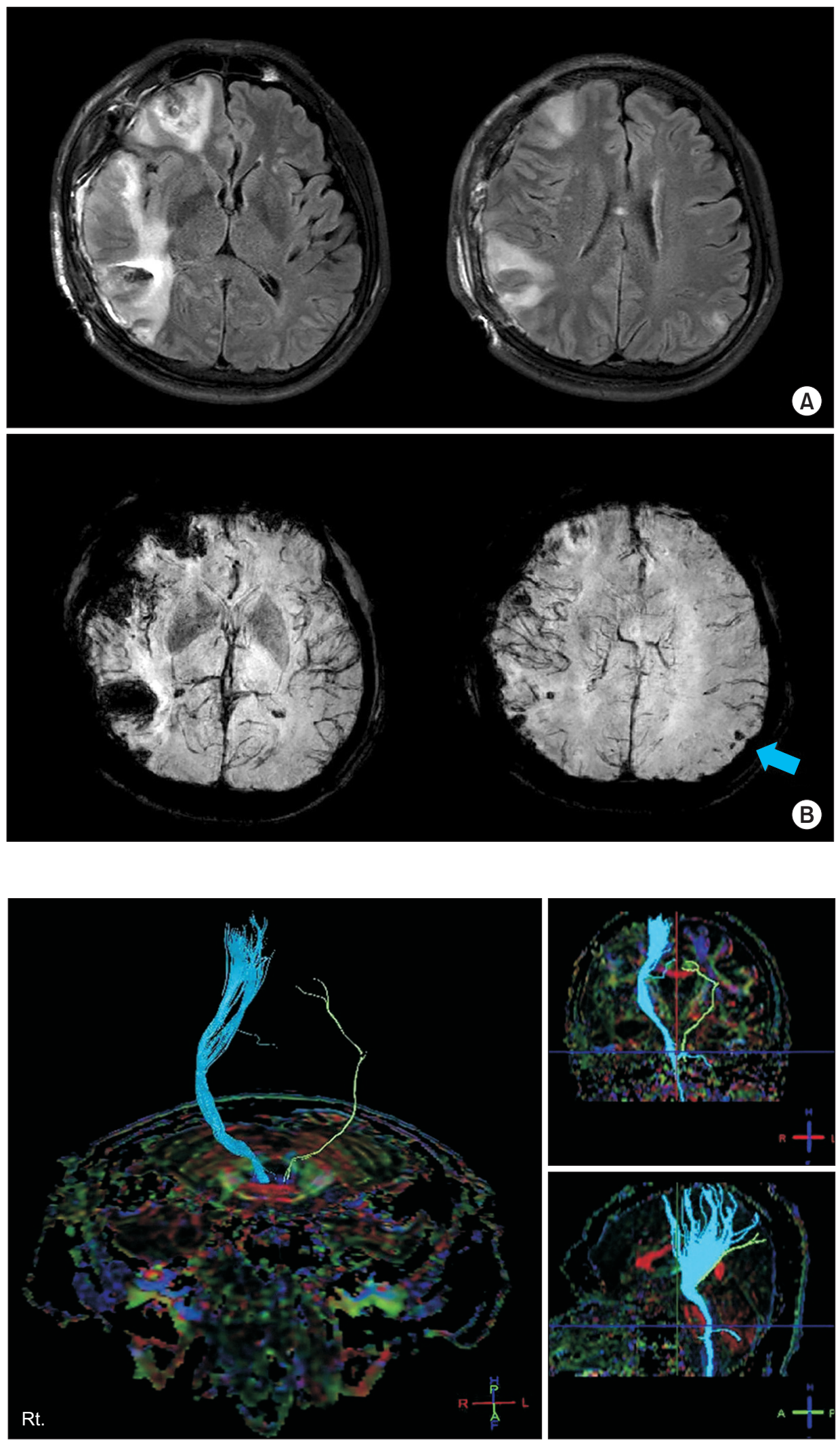

Fig. 2. Brain magnetic resonance imaging at 1 week after injury. (A) T2 FLAIR image (TR/TE=9000/120 $\mathrm{ms}$ ) shows cytotoxic edema with diffusion restriction in right inferior frontal and right temporal lobe. In addition, hemorrhagic cortical contusions in right inferior frontal, temporal, and left parietal lobe can be seen; otherwise, no definite abnormalities such as cerebral infarction were found in the left hemisphere. (B) Susceptibility-weighted image (TR/TE=22.8/31.9 ms) shows multiple microhemorrhages in left parietal and occipital subcortical regions (blue arrow).

Fig. 3. Diffusion tensor image at 3 months after injury shows that the right corticospinal tract (CST) was injured in the subcortical white matter and that the left CST was nearly completely injured. 
Table 1. Mean values of FA and ADC

\begin{tabular}{|c|c|c|c|c|c|c|}
\hline & \multicolumn{6}{|c|}{ Diffusion tensor image } \\
\hline & \multicolumn{2}{|c|}{ CST } & \multicolumn{2}{|c|}{ PLIC } & \multicolumn{2}{|c|}{ Medulla } \\
\hline & Left & Right & Left & Right & Left & Right \\
\hline FA & 0.418 & 0.495 & 0.437 & 0.492 & 0.502 & 0.502 \\
\hline $\operatorname{ADC}\left(\mathrm{mm}^{2} / \mathrm{s}\right)$ & 1.007 & 0.908 & 1.038 & 0.960 & 1.031 & 1.031 \\
\hline
\end{tabular}

CST, corticospinal tract; PLIC, posterior limb of internal capsule; FA, fraction anisotropy; ADC, apparent diffusion coefficients.

side. The FA and ADC values are shown in Table 1.

Motor-evoked potentials (MEPs) were measured at 2 months after the injury on abductor pollicis brevis (APB) muscles of both hands with an aim to enhance the results of DTT. A figure-of-eight shaped coil connected to a Magstim 200 magnetic stimulator (Magstim, Whitland, UK) was placed on scalp over the primary motor cortex (M1) for eliciting maximal MEPs in contralateral APB. Stimulation intensity for measuring MEP was set at the suprathreshold $\left(\mathrm{MT}_{1 \mathrm{mV}}\right)$ and MEP sizes in each muscle were determined by averaging MEPs with 15 stimuli [5]. Stimulation of the right M1 showed $1.35 \mathrm{mV}$ of MEP on left APB. In contrast, stimulating the left M1 showed no $\mathrm{MEP}$ responses on right $\mathrm{APB}$.

\section{DISCUSSION}

In this case study, we report a case of a patient with right hemiplegia after TBI whose initial brain CT showed brain damage mainly on the right side and in whom DTT showed complete injury to the left CST.

In the process of TBI, mechanical forces that shear axons can occur on the opposite site of impact by inertial as well as by direct forces at the site of the impact. DAI and focal contusion can result in neuronal disconnection, or diaschisis in TBI, which could induce motor weakness. Furthermore, transtentorial herniation can bring about CST injury by compressing the cerebral peduncle, which is caused by the downward displacement of medial brain structures out of the cranium through the tentorial notch following head trauma $[3,6]$. Because of these complex etiologies of TBI, it is difficult to recognize the actual neurologic origins of the symptoms in TBI patients. Since conventional MRI provides limited information about such etiologies, DTI has been used to demonstrate the axonal injuries after TBI [6].
In the present case, we performed MEP studies with an aim to enhance the results of DTI study. MEP is a useful measure for establishing the functional status of the CST, and it is widely used to predict the neurologic prognosis in many neurologic abnormalities including TBI [7]. However, MEP could not show the exact site of the injury of CST. For this reason, DTI is proposed as an additional study to clarify the location and extent of damage.

Brain physiology and neurology are still obscure, and many tools and techniques are currently being developed to reveal the pathophysiology of brain. Clinicians who treat brain injury patients can miss the chance of accurate evaluation if they depend on limited tools. In most cases of TBI, we prefer conventional brain imaging techniques, but DTI can provide additional information as well as help to localize pathologic lesions within the broad range of TBI.

In conclusion, we propose DTI as a possible and useful add-on study for patients with DAI after TBI whose clinical symptoms could not be explained by conventional imaging studies.

\section{CONFLICT OF INTEREST}

No potential conflict of interest relevant to this article was reported.

\section{REFERENCES}

1. Xu J, Rasmussen IA, Lagopoulos J, Haberg A. Diffuse axonal injury in severe traumatic brain injury visualized using high-resolution diffusion tensor imaging. J Neurotrauma 2007;24:753-65.

2. Nakayama N, Okumura A, Shinoda J, Yasokawa YT, Miwa K, Yoshimura SI, et al. Evidence for white matter disruption in traumatic brain injury without macroscopic lesions. J Neurol Neurosurg Psychiatr 2006;77: 850-5.

3. Yeo SS, Jang SH. Corticospinal tract recovery in a patient with traumatic transtentorial herniation. Neural Regen Res 2013;8:469-73.

4. Mori S, Zhang J. Principles of diffusion tensor imaging and its applications to basic neuroscience research. Neuron 2006;51:527-39.

5. Shin HW, Sohn YH. Interhemispheric transfer of paired associative stimulation-induced plasticity in 
the human motor cortex. Neuroreport 2011;22:166-70.

6. Seo JP, Jang SH. Traumatic axonal injury of the corticospinal tract in the subcortical white matter in patients with mild traumatic brain injury. Brain Inj 2015; 29:110-4.
7. Kim GW, Won YH, Park SH, Seo JH, Ko MH. Can motor evoked potentials be an objective parameter to assess extremity function at the acute or subacute stroke stage? Ann Rehabil Med 2015;39:253-61. 